\title{
Avaliação de serviços de saúde auditiva sob a perspectiva do usuário: proposta de instrumento
}

\section{Hearing health service evaluation under the perspective of the users: proposal of an instrument}

\author{
Maria Eliza Armigliato' ${ }^{1}$, Daniela Galvão de Almeida Prado ${ }^{2}$, Tatiana Mendes de Meloº \\ Maria Angelina Nardi de Souza Martinez ${ }^{4}$, Andréa Cintra Lopes ${ }^{5}$, \\ Regina Célia Bortoleto Amantini ${ }^{6}$, Maria Cecília Bevilacqua ${ }^{7}$
}

\begin{abstract}
RESUMO
Objetivo: Verificar a aplicabilidade de um questionário de avaliação do serviço, sob a perspectiva do usuário. Métodos: O questionário foi inspirado no estudo conduzido pelo Hearing and Communication Group, e apresenta 18 questões nas dimensões: acesso ao serviço, avaliação da audição, atendimento personalizado, benefício para família, comunicação e informação, e competência profissional. Cada questão foi apresentada em escala graduada de cinco pontos. A casuística foi composta por 53 pacientes (ou responsável/acompanhante), que foram convidados, pelos profissionais da recepção, a responder ao questionário. Os dados foram calculados em porcentagem e a pontuação de cada domínio e a pontuação total foi estabelecida. A estatística inferencial foi aplicada, adotando nível de significância de 5\%, a fim de verificar a influência das características demográficas no escore total. Resultados: O escore para o domínio acesso foi $62,25 \%$, para avaliação da audição foi $85,96 \%$, para atendimento personalizado foi $82,05 \%$, para benefício para família foi $68,57 \%$, para comunicação e informação foi 79,13\%, para competência profissional foi 97,39\% e para o escore total 70,65\%. Verificou-se que pacientes com menor nível de escolaridade, menor tempo de atendimento no serviço e os que já receberam o AASI apresentam resultados mais elevados no escore total do questionário. Conclusão: O questionário do presente estudo mostrou-se de fácil aplicabilidade no serviço de saúde auditiva, porém, é necessária sua aplicação em serviços de saúde auditiva em nível nacional para que a padronização e os critérios de confiabilidade e validade possam ser estabelecidos.
\end{abstract}

Descritores: Avaliação de serviços de saúde; Indicadores de qualidade em assistência à saúde; Sistema único de saúde; Audiologia; Perda auditiva

Trabalho realizado no Departamento de Fonoaudiologia da Faculdade de Odontologia de Bauru Universidade de São Paulo - USP - Bauru (SP), Brasil. (1) Acadêmica do Curso de Graduação em Fonoaudiologia da Faculdade de Odontologia de Bauru da Universidade de São Paulo - USP - Bauru (SP), Brasil. (2) Acadêmica do Curso de Graduação em Fonoaudiologia da Faculdade de Odontologia de Bauru da Universidade de São Paulo - USP - Bauru (SP), Brasil.

(3) Fonoaudióloga Especialista em Laboratório do Departamento de Fonoaudiologia da Faculdade de Odontologia de Bauru da Universidade de São Paulo - USP - Bauru (SP), Brasil.

(4) Doutora, Professora da Faculdade de Fonoaudiologia da Pontifícia Universidade Católica de São Paulo - PUC-SP - São Paulo (SP), Brasil.

(5) Doutora, Professora do Departamento de Fonoaudiologia da Faculdade de Odontologia de Bauru da Universidade de São Paulo - USP - Bauru (SP), Brasil.

(6) Doutora, Fonoaudióloga da Divisão de Saúde Auditiva do Hospital de Reabilitação de Anomalias Craniofaciais da Universidade de São Paulo USP - Bauru (SP), Brasil.

(7) Livre-Docente, Professora do Departamento de Fonoaudiologia da Faculdade de Odontologia de Bauru da Universidade de São Paulo - USP - Bauru (SP), Brasil.

Endereço para correspondência: Tatiana Mendes de Melo. Centro de Pesquisas Audiológicas. R. Silvio Marchione, 3-20, Bauru (SP), Brasil, CEP: 17012-900 (Caixa Postal 1501).E-mail: cpa@centrinho.usp.br

Recebido em: 17/4/2009; Aceito em: 29/6/2009

\section{INTRODUÇÃO}

A avaliação das ações de saúde vem ocupando lugar de destaque entre as ações de planejamento e gestão de práticas de saúde $^{(1)}$. No Brasil, diversas iniciativas voltadas para avaliação em saúde vêm sendo desenvolvidas de forma progressiva nas últimas décadas, tanto no âmbito das pesquisas acadêmicas, com vistas à avaliação de serviços de saúde, até a incorporação, pelo Ministério da Saúde, de pesquisas avaliativas com vistas a subsidiar a elaboração de políticas e programas setoriais e a difusão de seus resultados ${ }^{(2)}$.

De maneira geral, a avaliação da qualidade dos serviços de saúde segue os conceitos teóricos que aborda os três seguimentos: a estrutura, o processo e os resultados. A avaliação da estrutura corresponde às condições físicas, equipamentos, materiais e profissionais presentes no serviço de saúde, enquanto que a avaliação do processo corresponde ao conjunto de atividades desenvolvidas na relação profissional e paciente e a avaliação dos resultados refere-se às mudanças verificadas na condição de saúde do paciente ${ }^{(3)}$. 
Neste último item da tríade também está inserida a satisfação do usuário a partir do cuidado prestado, uma vez que não se pode ter um serviço de alta qualidade quando o alvo deste serviço - o usuário - não estiver satisfeito ${ }^{(4-5)}$. Os estudos sob este prisma ganharam destaque na literatura principalmente na década de 1970, nos Estados Unidos e na Inglaterra, como um atributo da qualidade. No Brasil, os estudos se desenvolveram na década de 1990, a partir do fortalecimento do controle social no âmbito do Sistema Único de Saúde (SUS), por intermédio da participação da comunidade nos processos de planejamento e avaliação dos serviços ${ }^{(6)}$.

O debate acerca de como é realizada a investigação da satisfação do paciente aponta basicamente duas naturezas metodológicas: a abordagem quantitativa e qualitativa. Os métodos quantitativos são os mais utilizados, em forma de questionários com questões fechadas, por serem de fácil manejo e de administração anônima, com baixo custo operacional. Geralmente os instrumentos reportados na literatura não apresentam padronização e critérios de validade e confiabilidade, principalmente quando aplicados em sistemas de saúde em que os itens de investigação não são transferíveis ou adaptáveis às condições de outros serviços ${ }^{(7-10)}$.

Embora ainda sejam incipientes as investigações sobre os fatores que determinam a satisfação do usuário, é sabido que muitos são relativos ao serviço, aos profissionais e até mesmo às características dos próprios pacientes. Em relação aos fatores inerentes ao serviço e aos profissionais, a cortesia da equipe, as informações fornecidas sobre o tratamento, a resolutibilidade, a disponibilidade e o tempo de espera do atendimento são os aspectos mais relevantes ${ }^{(11-14)}$. Por outro lado, o estado geral de saúde, os benefícios proporcionados pelo tratamento, a idade, o estado civil e o grau de escolaridade do paciente tendem a serem aspectos que interferem na avaliação da qualidade do serviço por parte do usuário ${ }^{(15-17)}$.

É importante ressaltar que na avaliação dos serviços de saúde de caráter público é bastante visível o viés da gratidão, que se trata da omissão de questionamentos e críticas negativas dos usuários, principalmente em países em desenvolvimento, tanto pelo medo de perder o acesso, quanto pela relação de dependência e afetividade com os profissionais de saúde ${ }^{(8,10,18)}$. Além disso, para certos segmentos da população, a dificuldade usual de conseguir atendimento resulta em baixa expectativa. O simples fato de ser atendido já pode produzir satisfação, o que não quer dizer que o serviço de saúde tenha atingido às expectativas do paciente ${ }^{(10,18)}$.

No âmbito da saúde auditiva, diante da estimativa do número de deficientes auditivos em nível internacional e nacional, faz-se necessária a otimização dos recursos financeiros empregados na intervenção audiológica ${ }^{(19)}$, a fim de possibilitar melhor benefício para esta população. Pacientes com alterações auditivas entram nos serviços de saúde auditiva com o objetivo de melhorar sua função auditiva e consequentemente seu desempenho comunicativo. Quando o tratamento é fornecido, como a concessão do aparelho de amplificação sonora individual (AASI) ou o serviço de reabilitação, ou os dois, a efetividade do tratamento precisa ser avaliada ${ }^{(20)}$.

A partir da implantação da Política de Atenção à Saúde Auditiva no Brasil, em 2004, muitos avanços foram conquistados com o intuito de beneficiar a população deficiente auditiva. A avaliação dos serviços de saúde auditiva vem como uma forma de acrescentar melhorias a esta política, a partir da otimização da verba pública para o atendimento de qualidade para um maior número de indivíduos com perda auditiva.

$\mathrm{Na}$ literatura referente à Audiologia, muito têm se estudado em relação ao benefício e satisfação do paciente em relação ao uso do AASI como forma de avaliar os resultados da intervenção. Os questionários de auto-avaliação são utilizados, mas a análise dos mesmos não é suficiente para avaliação da qualidade dos serviços de saúde auditiva sob a ótica de seus usuários.

Neste sentido, o presente estudo tem o intuito de verificar a aplicabilidade de um questionário de avaliação de serviço de saúde auditiva, sob a perspectiva do usuário, elaborado para ser aplicado nos serviços de saúde auditiva do SUS.

\section{MÉTODOS}

O presente estudo faz parte do projeto multicêntrico "Indicadores e proposta de avaliação da qualidade dos serviços de saúde auditiva do Sistema Único de Saúde", financiado pelo Conselho Nacional de Desenvolvimento Científico e Tecnológico (CNPq) e aprovado pelo Comitê de Ética em Pesquisa da Faculdade de Odontologia de Bauru da Universidade de São Paulo (processo no 092/2008). Toda investigação foi desenvolvida na Clínica de Fonoaudiologia da Faculdade de Odontologia de Bauru da Universidade de São Paulo (FOB-USP).

Embora os protocolos de avaliação de serviços de saúde auditiva sob a perspectiva do paciente, utilizados internacionalmente de maneira pontual, tenham sido analisados pelos pesquisadores do presente estudo, nenhum foi considerado adequado para as necessidades e realidade brasileira. Desta forma, para esta proposta foi elaborado um questionário, inspirado no estudo conduzido pelo Hearing and Communication Group $^{(21)}$, que contêm 18 perguntas, referentes às dimensões: acesso ao serviço (quatro questões), avaliação da audição (duas questões), atendimento personalizado (duas questões), benefício para família (duas questões), comunicação e informação (seis questões) e competência profissional (duas questões). As respostas para cada pergunta do questionário foram apresentadas no formato de múltipla escolha e, em uma escala graduada de cinco pontos. Além das dimensões avaliadas, as características demográficas do usuário foram abordadas no início do questionário (Anexo 1).

Precedendo a aplicação do presente estudo, o conteúdo do questionário foi analisado por juízes que realizaram ajustes finais, de ordem semântica, a fim de facilitar a compreensão do mesmo pela população atendida em serviços de saúde auditiva do SUS. Outros ajustes foram realizados após a condução de um estudo piloto com pacientes do serviço de saúde auditiva da instituição.

Desta forma, a casuística foi composta por 53 pacientes (ou responsável/acompanhante, caso o paciente fosse criança ou inapto para responder o questionário) do serviço de saúde auditiva da Clínica de Fonoaudiologia da instituição, de ambos os gêneros, atendidos nos meses de novembro e dezembro de 2008. 
A Clínica de Fonoaudiologia é credenciada pelo SUS como Centro de Referência em Alta Complexidade em Saúde Auditiva desde agosto de 2003, envolvendo atividades de prevenção, identificação, diagnóstico da deficiência auditiva, bem como indicação e adaptação do dispositivo eletrônico aplicado à surdez e a terapia fonoaudiológica. Este serviço de saúde auditiva confere cobertura para 68 cidades do interior, correspondendo a uma população de 1.623.027 habitantes e conta com amplo espaço físico, com salas equipadas para o atendimento de pessoas com distúrbios de audição nas diferentes faixas etárias. A equipe interdisciplinar é representada por 12 fonoaudiólogas, um médico otorrinolaringologista e um neuropediatra, uma assistente social, uma psicóloga (e mais três funcionários de ordem administrativa - faturamento/recepção).

O paciente, ao apresentar-se na recepção para o atendimento audiológico, foi informado sobre a existência do questionário de avaliação do serviço pelos funcionários deste setor, e convidado a preencher o instrumento após a finalização do atendimento no setor de saúde auditiva, não havendo restrição com relação ao gênero, escolaridade e características audiológicas. O mesmo foi orientado que o instrumento deveria ser preenchido por ele (ou seu acompanhante), sem auxílio dos profissionais do serviço. Ao finalizar, o paciente deveria colocar o questionário em uma urna fechada localizada na sala de espera da Clínica.

As respostas obtidas nos questionários foram tabuladas em planilha de dados e ao final a porcentagem de cada domínio e a pontuação total foi calculada como apresentado no Anexo 2. O resultado da pontuação total do questionário foi calculado somando-se o número total de pontos acumulados em cada questão, sendo possível a obtenção de no máximo 90 pontos. Essa pontuação foi transformada em porcentagem (regra de três), onde $100 \%$ é o escore máximo. Em cada domínio esta linha de raciocínio também foi seguida para o cálculo do escore, isto é, os pontos acumulados das questões do domínio foram somados e posteriormente transformados em porcentagem, sendo que cada domínio também apresentava como escore máximo $100 \%$.

A estatística inferencial foi aplicada comparando o escore total obtido com as características demográficas: idade, grau da dificuldade auditiva, escolaridade, tempo de atendimento no serviço de saúde auditiva, utilizando-se a Correlação de Spearman. O escore total e as variáveis: gênero, recebimento do AASI e atendimento em outros serviços de saúde auditiva foram analisados por meio do Teste t Student; enquanto que os itens referentes ao respondente do questionário e tipo de atendimento pretendido foram analisados com o escore total do protocolo por meio da Análise de Variância (ANOVA). Em todos os testes estatísticos utilizados foi adotado nível de significância de 5\%.

\section{RESULTADOS}

Inicialmente serão descritos os dados referentes às características demográficas dos pacientes que responderam o questionário proposto pelo presente estudo. Tais informações podem ser visualizadas na Tabela 1.

Como é possível observar o perfil dos respondentes desta pesquisa caracterizou-se por pacientes do gênero feminino,
Tabela 1. Dados demográficos dos participantes da pesquisa $(\mathrm{N}=53)$

\begin{tabular}{|c|c|}
\hline Variável & $\%$ \\
\hline \multicolumn{2}{|l|}{ Quem respondeu o questionário } \\
\hline Acompanhante & 30,19 \\
\hline Paciente & 49,06 \\
\hline Responsável & 13,21 \\
\hline Não respondeu & 7,55 \\
\hline \multicolumn{2}{|l|}{ Sexo } \\
\hline Feminino & 73,58 \\
\hline Masculino & 26,42 \\
\hline \multicolumn{2}{|l|}{ Idade } \\
\hline Até 15 anos & 2,00 \\
\hline 16 a 30 anos & 6,00 \\
\hline 31 a 45 anos & 18,00 \\
\hline 46 a 60 anos & 26,00 \\
\hline 61 a 75 anos & 36,00 \\
\hline Mais de 76 anos & 12,00 \\
\hline \multicolumn{2}{|l|}{ Grau da dificuldade auditiva } \\
\hline Não tenho dificuldade auditiva & 3,77 \\
\hline Leve & 3,77 \\
\hline Moderada & 30,19 \\
\hline Severa & 20,75 \\
\hline Profunda & 30,19 \\
\hline Sem resposta & 11,32 \\
\hline \multicolumn{2}{|l|}{ Escolaridade } \\
\hline Analfabeto & 9,43 \\
\hline Alfabetizado & 26,42 \\
\hline $1^{\circ} \mathrm{grau}$ & 33,96 \\
\hline Ensino médio & 20,75 \\
\hline Ensino superior & 3,77 \\
\hline Sem resposta & 5,66 \\
\hline \multicolumn{2}{|l|}{ Tipo de atendimento buscado } \\
\hline Exames de audição & 28,30 \\
\hline Aparelho auditivo & 64,15 \\
\hline Terapia fonoaudiologica & 3,77 \\
\hline Outros serviços & 0,00 \\
\hline Sem resposta & 3,77 \\
\hline \multicolumn{2}{|l|}{ Já recebeu aparelho auditivo? } \\
\hline Sim & 66,04 \\
\hline Não & 26,42 \\
\hline Sem resposta & 7,55 \\
\hline \multicolumn{2}{|c|}{ Já foi atendido em outros serviços de saúde auditiva? } \\
\hline Sim & 37,74 \\
\hline Não & 56,60 \\
\hline Sem resposta & 5,66 \\
\hline \multicolumn{2}{|c|}{ Há quanto tempo é atendido neste serviço } \\
\hline Mais de um ano & 60,38 \\
\hline Entre 6 meses e 1 ano & 20,75 \\
\hline Entre 3 meses e 6 meses & 9,43 \\
\hline Entre 1 mês e 3 meses & 7,55 \\
\hline Menos de 1 mês & 0,00 \\
\hline Sem resposta & 1,89 \\
\hline
\end{tabular}

com idade entre 61 e 75 anos, com grau de dificuldade auditiva de moderada a profunda, com nível de escolaridade até $1^{\circ}$ grau e que procuraram o serviço em busca do aparelho auditivo. Destes, 66,04 \% já tinham recebido o AASI, 56,60\% não tinham sido atendidos em outros serviços de saúde auditiva 
e eram pacientes da instituição a mais de um ano (60,78\%).

De acordo com os resultados obtidos dos escores dos domínios e da pontuação do questionário, conforme apresentado na Figura 1, verifica-se que o item acesso apresenta o pior resultado entre os domínios, enquanto que o melhor índice obtido refere-se à competência profissional.

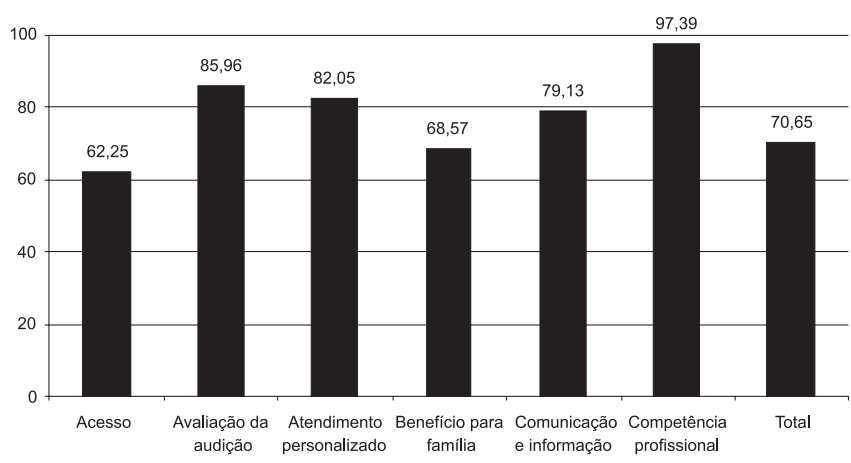

Figura 1. Resultados dos escores para cada domínio do questionário $(\mathrm{N}=53)$

Para apurar a influência das características demográficas dos pacientes no resultado final do questionário foi aplicada a análise estatística inferencial. A partir dos resultados apresentados na Tabela 2 constata-se que a escolaridade, tempo de atendimento no serviço de saúde auditiva e recebimento do AASI são variáveis que interferem, de forma estatisticamente significante, no resultado obtido no escore total do instrumento, ou seja, os pacientes com maior nível de escolaridade, com maior tempo de atendimento no serviço e os que ainda não tinham recebido o AASI apresentam resultados inferiores no escore total do questionário, comparado aos pacientes com menor nível de escolaridade, com menor tempo de atendimento no serviço e os que já havia recebido o AASI.

Uma observação que deve ser relatada refere-se ao preenchimento das informações, isto é, respostas referentes às características demográficas e sobre a avaliação do serviço

Tabela 2. Características demográficas e escore total do questionário

\begin{tabular}{|c|c|c|}
\hline Teste estatístico & Variáveis & Valor de $p$ \\
\hline \multirow[t]{3}{*}{ t de Student } & Sexo & 0,682 \\
\hline & $\begin{array}{l}\text { Já recebeu aparelho } \\
\text { auditivo }\end{array}$ & $0,001^{*}$ \\
\hline & $\begin{array}{l}\text { Já foi atendido em } \\
\text { outros serviços }\end{array}$ & 0,053 \\
\hline \multirow[t]{2}{*}{ Análise de variância } & $\begin{array}{l}\text { Tipo de atendimento } \\
\text { buscado }\end{array}$ & 0,177 \\
\hline & $\begin{array}{l}\text { Quem respondeu o } \\
\text { questionário }\end{array}$ & 0,237 \\
\hline Correlação de & Idade & $0,116(r=-0,23)$ \\
\hline \multirow[t]{3}{*}{ Pearson } & Escolaridade & $0,040^{*}(r=-0,28)$ \\
\hline & $\begin{array}{l}\text { Grau de dificuldade } \\
\text { auditiva }\end{array}$ & $0,061(r=-0,30)$ \\
\hline & $\begin{array}{l}\text { Há quanto tempo é } \\
\text { atendido no serviço }\end{array}$ & $0,022^{*}(r=-0,32)$ \\
\hline
\end{tabular}

* Valores estatisticamente significantes $(p<0,05)$ não foram preenchidas pelos respondentes, como também houve a assinalação de mais de uma resposta para uma única questão. Contudo, tais fatos não proporcionaram nenhum tipo de prejuízo cientifico na apreciação dos dados do estudo, pois a análise considerou somente as questões preenchidas de forma adequada (uma opção por questão).

Outro ponto que deve ser enfatizado, mesmo tratando-se de resultados qualitativos da aplicação do questionário, é referente ao discurso dos profissionais da recepção, que relataram a recusa de alguns pacientes em responder ao instrumento, ora por não saber ler, ora por não compreender algumas questões e precisar de outra pessoa para auxiliar o preenchimento do instrumento.

\section{DISCUSSÃO}

Estudos referentes à avaliação da qualidade de serviços do SUS sob a ótica dos usuários têm sido amplamente relatados na literatura em diversas áreas da saúde ${ }^{(4-5,7,10-11,13-15,18)}$, contudo, pesquisas sobre esta temática nos serviços de saúde auditiva do país são escassas, uma vez que o atendimento integral do indivíduo deficiente auditivo foi implantado pelo Ministério da Saúde a partir de setembro de 2004, com a instalação da Política Nacional de Atenção a Saúde Auditiva. Atualmente há algumas experiências pontuais e o esforço de alguns gestores para que a avaliação da qualidade destes serviços comece acontecer no Brasil.

A partir dos resultados obtidos foi possível identificar escores elevados na pontuação total e por domínios do questionário, indicando que os respondentes do presente estudo avaliaram de forma positiva o atendimento prestado na Instituição. Os domínios competência profissional, avaliação da audição e atendimento personalizado aparecem com melhores escores $(97,39 \%, 85,96 \%$ e $82,05 \%$, respectivamente), enquanto que os domínios acesso e beneficio para família apresentam os piores escores $(62,25 \%$ e $68,57 \%$, respectivamente).

Apesar dos índices elevados de satisfação apresentados pelas dimensões do questionário, estes dados apresentam uma tendência peculiar, ou seja, os resultados obtidos podem ser reflexos da dificuldade de argumentação dos usuários do SUS (que geralmente são pacientes com menor nível educacional), como também da relutância em expressar críticas e opiniões negativas (viés da gratidão) e o próprio receio dos pacientes em perder o direito ao atendimento prestado ${ }^{(8,10,18)}$. No entanto, estas limitações são inerentes a estudos de qualquer área da saúde com o intuito de verificar a satisfação dos pacientes ao atendimento prestado no SUS e não devem ser desconsiderados; necessitam, somente, ser analisados com cautela.

Cabe ressaltar que, após a aplicação do questionário e discussões com especialistas, uma modificação estrutural foi sugerida em relação à oitava pergunta do questionário, que deve ser reformulada, para pesquisas futuras, da seguinte maneira "As decisões ou opções quanto ao tipo de tratamento foram discutidos com você e com a família? (exemplo: aparelho auditivo, molde, escola, trabalho ou outros)". Como a dimensão do beneficio para família foi visualizado como um tópico de difícil interpretação na avaliação da qualidade do serviço de saúde auditiva em função da sua subjetividade, 
sugere-se a retirada deste domínio em estudos posteriores que utilizarão o questionário desenvolvido para o presente trabalho.

Ao avaliar a influência das características individuais no escore obtido na pontuação total do trabalho constatou-se que a escolaridade do respondente interfere, de forma estatisticamente significante, no resultado obtido, isto é, pacientes com menor nível de instrução tendem a apresentar respostas mais positivas que os demais respondentes do questionário. Por outro lado, a variável idade, que geralmente é apresentada como fator influente na avaliação da qualidade do serviço por parte do usuário em outros trabalhos desenvolvidos em serviços de saúde ${ }^{(15-17)}$, no presente trabalho, não influenciou os resultados obtidos na pontuação total do questionário (Tabela 2).

Por outro lado, em relação às características do serviço, o tempo de atendimento no serviço de saúde auditiva e recebimento do AASI foram fatores que também interferiram, de forma estatisticamente significante, no resultado obtido no escore total do instrumento (Tabela 2).

Considerando que $64,15 \%$ dos respondentes buscavam o fornecimento do AASI (Tabela 1), não é admirável que o recebimento de AASI seja um fator que interfira no escore total do questionário. A resolutibilidade do atendimento, que para os pacientes com perda de audição é sintetizado na adaptação do aparelho auditivo, é apontada como um dos fatores, em relação ao serviço, que mais influencia as respostas dos pacientes ${ }^{(12)}$.

Ao analisar as dificuldades assinaladas em relação à aplicação do questionário (preenchimento das informações e aceitação do paciente em responder o instrumento) é possível cogitar que tais situações podem ser decorrentes da idade (que em $48 \%$ dos casos é acima dos 61 anos), como também do nível educacional dos respondentes $(69,81 \%$ completaram até o ensino fundamental). Entretanto, são fatos que devem ser levados em consideração em estudos posteriores, uma vez que a população idosa do país cresce gradativamente e que o SUS atende, de forma predominante, pacientes com menor nível educacional.

Desta maneira, este trabalho apresenta uma proposta inicial de avaliação do serviço de saúde auditiva sob a ótica dos pacientes, com o desenvolvimento e aplicação do questionário em um único serviço no Estado de São Paulo. O estudo indica uma tendência de satisfação geral nos domínios superiores a $70 \%$ de escore, e no futuro, talvez isto possa ser considerado como um indicador de qualidade do serviço.

Neste momento torna-se indispensável a continuidade do estudo, com a aplicação do instrumento em serviços de diferentes regiões do país. Este prosseguimento será trabalhado no projeto multicêntrico "Indicadores e proposta de avaliação da qualidade dos serviços de saúde auditiva do Sistema Único de Saúde" (CNPq), a fim de padronizar, validar e desenvolver as características psicométricas do instrumento ${ }^{(7-10)}$, bem como estabelecer a pontuação de corte do escore deste questionário que possa ser um indicador de qualidade do serviço, para que futuramente, esta proposta seja apresentada para o Ministério da Saúde e ser formalizada para os 128 serviços de saúde auditiva do SUS.

\section{CONCLUSÃO}

O questionário desenvolvido para o presente estudo, inspirado no trabalho conduzido pelo Hearing and Communication Group, mostrou-se de fácil aplicabilidade no serviço de saúde auditiva, mesmo com as limitações apresentadas, que são passiveis de ocorrerem em qualquer estudo de avaliação do serviço de saúde do SUS pelos seus usuários.

Neste momento, o instrumento será aplicado em diferentes serviços do país, retirando-se o domínio benefício para família e ajustando a oitava pergunta do questionário, a fim de padronizá-lo e validá-lo para realidade brasileira, para que, em um futuro não distante, seja sugerida ao Ministério da Saúde a sua utilização nos serviços de saúde auditiva do SUS, como uma forma de avaliação da qualidade dos mesmos.

\section{AGRADECIMENTOS}

Ao Conselho Nacional de Desenvolvimento Científico e Tecnológico (CNPq), pelo financiamento concedido para realização desta pesquisa por meio de bolsa de iniciação científica (processo número 502837/2007-1).

\begin{abstract}
Purpose: To verify the applicability of a questionnaire for the assessment of hearing health services, under the perspective of the users. Methods: The questionnaire was inspired on the study conducted by the Hearing and Communication Group, and presents 18 questions focused on the following dimensions: access to the service, hearing evaluation, personal attendance, family benefits, communication and information, and professional competence. Each question of this instrument was presented in a graduation scale of five points. The casuistic comprised 53 patients (or caregiver), that were invited, by the reception professionals, to answer to the questionnaire. Data were calculated in percentage, and the score for each domain and the total score were established. Inferential statistic tests were applied, adopting a significant level of 5\%, in order to verify the influences of demographic characteristics on the total score. Results: The score for the access domain was $62.25 \%$, for hearing evaluation was $85.96 \%$, for personal attendance was $82.05 \%$, for family benefits was $68.57 \%$, for the domain communication and information was $79.13 \%$, for professional competence was $97.39 \%$, and the total score was $70.65 \%$. It was verified that patients with lower schooling levels, lesser time attending the service, and those that had already received the hearing aid obtained higher total scores on the questionnaire. Conclusion: The questionnaire used in the present study proved to be easily applicable in hearing health services; however, its application on national level is necessary, so that standardization and reliability and validity can be established.
\end{abstract}

Keywords: Health services evaluation/quality indicators; Single health system; Audiology; Hearing loss 


\section{REFERÊNCIAS}

1. Bosi ML, Uchimura KY. Avaliação da qualidade ou avaliação qualitativa do cuidado em saúde? Rev Saúde Publica. 2007;41(1):150-3.

2. Felisberto E. Da teoria à formulação de uma política nacional de avaliação em saúde: reabrindo o debate. Ciênc Saúde Coletiva. 2006;11(3):553-63.

3. Donabedian A. The definition of quality and approaches to its assessment. Ann Arbor (MI): Mich Health Administration Press;1980. [Explorations in quality assessment and monitoring, v.1]

4. Akerman M, Nadanovsky P. Avaliação dos serviços de saúde - avaliar o quê? Cad Saúde Publ. 1992;8(4):361-5.

5. Santos MP. Avaliação da qualidade dos serviços públicos de atenção a saúde da criança sob a ótica do usuário. Rev Bras Enferm. 1995;48(2):109-19.

6. Turris SA. Unpacking the concept of patient satisfaction: a feminist analysis. J Adv Nurs. 2005;50(3):293-8.

7. Lemme AC, Noronha G, Resende JB. A satisfação do usuário em hospital universitário. Rev Saúde Publ. 1991;25(1):41-6.

8. Jenkinson C, Coulter A, Bruster S, Richards N, Chandola T. Patients' experiences and satisfaction with health care: results of a questionnaire study of specific aspects of care. Qual Saf Health Care. 2002;11(4):3359.

9. Wensing M; Elwyn G. Research on patients' views in the evaluation and improvement of quality of care. Qual Saf Health Care. 2002;11(2):1537.

10. Esperidião M Trad LA. Avaliação de satisfação de usuários. Ciênc Saúde Coletiva. 2005;10(suppl):303-12.

11. Kloetzel K, Bertoni AM, Irazoqui MC, Campos VP, Santos RN. Controle de qualidade em atenção primária em saúde. I - A satisfação do usuário. Cad Saúde Publica. 1998;14(3):623-8.
12. Bernhart MH, Wiadnyana IG, Wihardjo H, Pohan I. Patient satisfaction in developing countries. Soc Sci Med. 1999;48(8):989-96.

13. Fonseca SM, Gutiérrez MG, Adami NP. Avaliação da satisfação de pacientes oncológicos com atendimento recebido durante o tratamento antineoplásico ambulatorial. Rev Bras Enferm. 2006;59(5):656-60.

14. Pietrovsk V, Dall’Agnol CM. Situações significativas no espaço-contexto da hemodiálise: o que dizem os usuários de um serviço? Rev Bras Enferm. 2006;59(5):630-5.

15. Souza EM, Pereira MG. A satisfação dos usuários na avaliação dos serviços de saúde. Brasília Méd. 1999;36(1/2):33-6.

16. Jackson JL, Chamberlin J, Kroenke K. Predictors of patient satisfaction. Soc Sci Med. 2001;52(4):609-20.

17. Seclén-Palacin J, Darras C. Satisfacción de los usuarios de salud: factores sociodemográficos y de accesibilidad asociados. An Fac Med (Peru). 2005;66(2):127-41.

18. Vaitsman J, Andrade GR. Satisfação e responsividade: formas de medir a qualidade e humanização da assistência à saúde. Ciênc Saúde Coletiva. 2005;10(3):599-613.

19. Gatehouse $\mathrm{S}$. The impact of measurement goals on design specification for outcomes measures. Ear Hear.(Suppl). 2000;21(4 Suppl):100S-105S.

20. Cook JA, Hawkins DB. Outcome measurement for patients receiving hearing aid services. Laryngoscope. 2007;117(4):610-3.

21. The Scottish Government. Draft quality standarts for adult hearing rehabilitation [Internet] 2008 Jan [cited 2008 Sep 16]. Available from: http://www.scotlandd.gov.uk/publications/2008/01/09093543/0.

Anexo 1. Instrumento de avaliação do serviço de saúde auditiva

Para oferecer melhores serviços à comunidade, a Clínica de Fonoaudiologia está conduzindo esta pesquisa entre os usuários de nossa clínica. O nosso objetivo é identificar onde podemos melhorar. Esperamos que você possa nos ajudar a reconhecer as áreas mais carentes em nossa clínica para que possamos oferecer melhor assistência. Você poderá apresentar comentários sobre quaisquer itens que, em sua opinião, poderiam beneficiar os nossos usuários. Você ou suas respostas não serão identificadas.

Queira, por gentileza, devolver as respostas a este questionário na caixa localizada na sala de espera do serviço.

Enquanto isso, caso deseje mais informações, entre em contato comigo pelo (telefone). Não é necessário se identificar. Será um prazer falar com você. A sua opinião é muito importante para nós. Agradeço a sua atenção e o tempo que levou para responder a este questionário.

Inicialmente gostaríamos de saber um pouco de você:
Paciente ( )
Responsável ( )
Acompanhante ( )
Sexo: Feminino ( ) Masculino ( ) Idade:

Como você descreve o grau da sua dificuldade auditiva ou de seu filho?
(1) não tenho dificuldade auditiva
(2) leve
(3) moderada
(4) severa
(5) profunda

Sua escolaridade
(1) analfabeto
(2) alfabetizado
(3) $1^{\circ}$ grau
(4) ensino médio
(5) ensino superior

Como você chegou aqui na Clínica de Fonoaudiologia?

(1) transporte fornecido

(2) transporte com recursos próprios

A consulta foi agendada diretamente após a primeira solicitação ou foi necessário passar por outras avaliações para, então, ser encaminhado para cá.

$\square$ Sim, foi marcada diretamente;

$\square$ Não, foram marcadas consultas de médico otorrinolaringologista (médico de ouvido) em outros lugares;

$\square$ Não, foram marcados exames de audição em outros lugares. 
O que você acha que deveria melhorar aqui na Clínica de Fonoaudiologia?

Que tipo de atendimento buscava?
(1) exames de audição
(2) aparelho auditivo
(3) terapia fonoaudiológica
(4) outros serviços
Quais:

1 - Você considera o acesso a Clínica de Fonoaudiologia:
(1) muito difícil
(2) difícil
(3) mais ou menos
(4) fácil
(5) muito fácil

2 - Tempo de espera para o primeiro atendimento/agendamento?
(1) mais de 1 ano
(2) entre 6 meses e 1 ano
(3) entre 3 meses e 6 meses
(4) entre 1 mês e 3 meses
(5) menos de 1 mês

3 - Após o primeiro atendimento, quanto tempo foi necessário para concluir seu exame de audição ou de seu filho?
(5) menos de 1 mês
(4) entre 1 mês e 3 meses
(3) entre 3 meses e 6 meses
(2) entre 6 meses e 1 ano
(1) mais de 1 ano

4 - Após o primeiro atendimento, quanto tempo foi necessário para receber o seu aparelho auditivo ou de seu filho?
(5) menos de 1 mês
(4) entre 1 mês e 3 meses
(3) entre 3 meses e 6 meses
(2) entre 6 meses e 1 ano
(1) mais de 1 ano

5 - Você recebeu explicação de cada exame realizado para avaliar sua audição ou de seu filho(a) e sobre os resultados?
(1) nunca
(2) raramente
(3) às vezes
(4) muitas vezes
(5) sempre

6 - Você recebeu explicação sobre os diferentes tipos de tratamentos possíveis e sobre o aparelho auditivo?
(1) nunca
(2) raramente
(3) às vezes
(4) muitas vezes
(5) sempre

7 - Você acha que os exames, consultas e retornos foram agendados e selecionados de acordo com as suas necessidades ou do seu filho?
(1) nunca
(2) raramente
(3) às vezes
(4) muitas vezes
(5) sempre

8 - As decisões quanto ao tipo de tratamento, aparelho auditivo, molde, escola, trabalho ou outros tratamentos foram discutidos com você e com a família?
(1) nunca
(2) raramente
(3) às vezes
(4) muitas vezes
(5) sempre

9 - Você acha que o tratamento melhorou o seu relacionamento com sua família ou com seu filho?
(1) não
(2) muito pouco
(3) muito
(4) bastante
(5) totalmente

10 - Você acha que o tratamento melhorou o seu relacionamento no seu trabalho e/ou escola?
(1) não
(2) muito pouco
(3) muito
(4) bastante
(5) totalmente

11 - Você teve oportunidade de esclarecer suas dúvidas sobre a audição, os exames e o tratamento seu ou de seu filho?
(1) nunca
(2) raramente
(3) às vezes
(4) muitas vezes
(5) sempre

12 - A família e os acompanhantes são convidados a participar no tratamento e recebem apoio da equipe de profissionais?
(1) nunca
(2) raramente
(3) às vezes
(4) muitas vezes
(5) sempre

13 - Como você avalia a informação que recebeu dos profissionais da Clínica de Fonoaudiologia?
(1) muito pouca ou ruim
(2) pouca ou ruim
(3) mais ou menos
(4) boa e esclarecedora
(5) muito boa, esclarecedora e motivadora

14 - Você recebeu por escrito a orientação sobre os exames de audição, aparelhos auditivos e tratamento?
(1) não
(2) sim, muito ruim
(3) sim, de difícil
(4) sim, boa e esclarecedora
(5) sim, muito boa, clara e de compreensão
fácil compreensão

15 - Você recebeu por escrito a orientação sobre as consultas agendadas?
(1) não
(2) sim, muito ruim
(3) sim, de difícil
(4) sim, boa e esclarecedora
(5) sim, muito boa, clara e de compreensão
fácil compreensão

16 - Você acha que estas informações devem ser por escrito?
(1) nunca
(2) raramente
(3) às vezes
(4) muitas vezes
(5) sempre

17 - A equipe da recepção é competente, interessada e atenciosa?
(1) não
(2) poucos
(3) vários
(4) a maioria
(5) todos

18 - Os profissionais são competentes, interessados e atenciosos?
(1) não
(2) poucos
(3) vários
(4) a maioria
(5) todos

19 - Você indicaria este centro para alguém?

(Não) Por quê?

(Sim) Por quê?

20 - O que você acha que deveria melhorar no seu atendimento ou no do seu filho? 
Anexo 2. Fórmula para o cálculo, em porcentagem, dos escores dos domínios e pontuação total do questionário

\begin{tabular}{|l|l|}
\hline Escore total & $1+2+3+4+5+6+7+8+9+10+11+12+13+14+15+16+17+18 / 90 * 100$ \\
\hline Escore - Acesso & $1+2+3+4 / 20 * 100$ \\
\hline Escore - Avaliação da audição & $5+6 / 10 * 100$ \\
\hline Escore - Atendimento personalizado & $7+8 / 10 * 100$ \\
\hline Escore - Benefício para família & $9+10 / 10 * 100$ \\
\hline Escore - Comunicação e informação & $11+12+13+14+15+16 / 30 * 100$ \\
\hline Escore - Competência profissional & $17+18 / 10 * 100$ \\
\hline
\end{tabular}

